\title{
Evaluación de los niveles de dependencia de la nicotina en usuarios de cigarrillos electrónicos
}

\section{Evaluating nicotine dependence levels in e-cigarette users}

\author{
Alba González-Roz*; Roberto Secades-Villa*; Sara Weidberg*. \\ *Departamento de Psicología. Universidad de Oviedo (España).
}

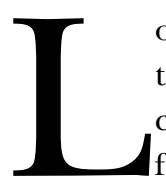
os cigarrillos electrónicos son dispositivos electrónicos de liberación de nicotina (DELN) incombustibles que mimetizan la experiencia de fumar tabaco (Harrell, Simmons, Correa, Padhya, y Brandon, 2014). Constan de tres elementos principales: una batería recargable, un atomizador y un cartucho.

Desde su introducción al mercado en el 2003, su prominencia y uso han incrementado notablemente a nivel mundial (Martínez-Sánchez et al., 2014). Los datos recientes sobre la prevalencia de los cigarrillos electrónicos en muestras europeas y americanas indican que, hasta la fecha, casi el 6,8\% de adultos usa los cigarrillos electrónicos (McMillen, Gottlieb, Shaefer, Winickoff, y Klein, 2015). No obstante, esta popularidad creciente ha generado un debate en la salud pública respecto de su seguridad y efectividad como alternativa de cesación tabáquica (Yu et al., 2016). Estudios sobre los cigarrillos electrónicos y la cesación tabáquica han ofrecido resultados mixtos. Mientras que las encuestas realizadas por Internet y los diseños no controlados sugieren que los cigarrillos electrónicos pueden ser efectivos para promover la abstinencia de tabaco, otros no han obtenido los mismos resultados. En la actualidad, las recomendaciones de la Organización Mundial de la Salud señalan que la efectividad de los cigarrillos electrónicos como método para dejar de fumar es limitada y requiere de mayor estudio (Organización Mundial de la Salud, 2014).
Otra preocupación involucra la propensión al abuso entre los usuarios actuales del cigarrillo electrónico (Cobb, Hendricks, y Eissenberg, 2015). La evidencia reciente muestra que los niveles de nicotina medidos en plasma y en saliva son similares a los de fumadores e incluso más altos que los niveles observados en usuarios de terapia de sustitución de nicotina (Marsot y Simon, 2015). Es más, los niveles bajos de dependencia de la nicotina y el incremento en el número de minutos entre el despertar y el primer uso autoinformados por los usuarios de cigarrillos electrónicos explican los niveles menores de dependencia de la nicotina en este grupo, en comparación con fumadores de tabaco (Foulds et al., 2015). El objetivo de este estudio es explorar los niveles de dependencia de la nicotina en una muestra de usuarios experimentados con el uso de cigarrillos electrónicos $(\mathrm{n}=39$; hombres $=77 \%)$ y comparar dichos resultados con fumadores de tabaco ( $\mathrm{n}=42$; hombres $=57 \%)$. Entre los usuarios de cigarrillos electrónicos, el 20,5\% usaban dispositivos de segunda generación y el $79,5 \%$ usaban dispositivos de tercera generación. Realizamos varias entrevistas presenciales con el fin de valorar factores sociodemográficos y de dependencia en ambos usuarios: de cigarrillos electrónicos y de tabaco. Usamos versiones adaptadas del Test de Fagerström de dependencia de la nicotina (FTND; Heatherton, Kozlowski, Frecker, y Fagerstrom, 1991) y la Escala del Síndrome de Dependencia de la Nicotina (NDSS; Shiffman, Waters, y Hickcox,

Recibido: Noviembre 2016; Aceptado: Noviembre 2016.

Enviar correspondencia a:

Alba González-Roz. Unidad Clínica de Conductas Adictivas, Departamento de Psicología Universidad de Oviedo, Plaza Feijóo, s/n, 33003 Oviedo, España. Fax: +3498510414. E-mail: albagroz@cop.es. 
2004) para analizar la dependencia de la nicotina en cada grupo. También recogimos marcadores bioquímicos de monóxido de carbono y un análisis de cotinina en orina. Nuestros hallazgos amplían estudios anteriores sobre el uso de cigarrillos electrónicos y la adicción a la nicotina. Dos descubrimientos principales surgieron de este estudio: (1) los usuarios de cigarrillos electrónicos tenían dependencia de los e-líquidos que contenían nicotina, y (2) la dependencia de la nicotina era menor entre los usuarios de cigarrillos electrónicos, comparado con fumadores actuales de tabaco (Tabla 1).

Varios mecanismos pueden explicar estos resultados. Primero, dado que la dependencia de la nicotina y la propensión al abuso están influenciados por la biodisponibilidad de nicotina, la ruta oral en la administración del e-líquido puede haber ejercido una influencia importante sobre las tasas de absorción y de exposición a la nicotina; por lo tanto, esto puede explicar los niveles de dependencia de la nicotina autoinformados por los usuarios de cigarrillos electrónicos. Segundo, además de la nicotina en sí, el uso de cigarrillos electrónicos también ha mostrado la implicación de otros componentes psicosociales que podrían explicar la adictividad entre los usuarios de cigarrillos electrónicos. Por ejemplo, el uso del cigarrillo electrónico permite al usuario sustituir gran parte de los componentes sensomotores y sociales asociados a fumar, tales como el ritual de llevarse la mano a la boca o la visualización del vapor al exhalar. Tercero, dado que la muestra de usuarios de cigarrillos electrónicos había sido fumadora con anterioridad, es posible que los participantes ya fueran dependientes de la nicotina del tabaco cuando iniciaron su uso de cigarrillos electrónicos. De esta manera, la nico- tina suministrada mediante el cigarrillo electrónico quizás mantenga la dependencia de la nicotina.

Este estudio no está exento de limitaciones. Primero, no se usaron versiones validadas de las escalas de dependencia administradas. De todas maneras, no pudimos realizar una prueba de validación porque el número de usuarios de cigarrillos electrónicos en la muestra captado durante el proceso de reclutamiento fue insuficiente para este fin. De todos modos, los datos sugieren que el uso del cigarrillo electrónico en España es probablemente menos prevalente que su uso en otros países europeos y en los EE.UU. Además, el hecho de que el 35,9\% de los usuarios de cigarrillos electrónicos también era fumador cuando los entrevistamos, impide que podamos ofrecer conclusiones sólidas en relación a los niveles de dependencia de la nicotina.

A pesar de estas limitaciones, dichos resultados suponen una contribución importante a nuestro conocimiento de la propensión al abuso entre los usuarios de cigarrillos electrónicos. Aunque los usuarios de cigarrillos electrónicos tenían dependencia de la nicotina, las medidas bioquímicas de monóxido de carbono y los cuestionarios autoinformados indicaron que la dependencia de la nicotina era menor entre los usuarios de cigarrillos electrónicos, comparada con la de los fumadores de tabaco. Se requieren estudios longitudinales que permitan determinar los niveles de dependencia de los usuarios de cigarrillos electrónicos. Por lo tanto, estudios futuros han de centrar su atención en explorar los niveles de dependencia de la nicotina en los usuarios de cigarrillos electrónicos, usando cuestionarios adaptados y validados para su medición.

Tabla 1. Niveles de dependencia entre usuarios de cigarrillos electrónicos y fumadores de tabaco

\begin{tabular}{|c|c|c|c|c|c|}
\hline & $\begin{array}{r}\text { Usuarios de } \\
\text { cigarrillos electrónicos } \\
(n=39)\end{array}$ & $\begin{array}{l}\text { Fumadores } \\
\qquad(n=42)\end{array}$ & $t$ & $p$ & $r$ \\
\hline $\mathrm{CO}(\mathrm{ppm})$ & $8 \pm 6,77$ & $15,24 \pm 7,18$ & $-4,657$ & $<, 001$ & 0,49 \\
\hline Cotinina $(\mathrm{ng} / \mathrm{mL})$ & $1891,26 \pm 1452,11$ & $2383,51 \pm 1129,07$ & $-1,710$ & ,091 & 0,16 \\
\hline FTND (tabaco/cigarrillo electrónico)a & $4,38 \pm 1,93$ & $5,57 \pm 1,48$ & $-3,118$ &, 003 & ,74 \\
\hline NDSS-T (tabaco/cigarrillo electrónico) ${ }^{a}$ & $26,26 \pm 5,29$ & $40,50 \pm 8,14$ & $-9,405$ & $<, 001$ & ,75 \\
\hline NDSS-Impulso (tabaco/cigarrillo electrónico)a & $10,46 \pm 4,72$ & $19,98 \pm 5,14$ & $-8,659$ & $<, 001$ & ,70 \\
\hline NDSS-Prioridad (tabaco/cigarrillo electrónico) ${ }^{a}$ & $4,82 \pm 1,57$ & $7,81 \pm 3,07$ & $-5,452$ & $<, 001$ &, 52 \\
\hline NDSS-Tolerancia (tabaco/cigarrillo electrónico)a & $14 \pm 3,41$ & $22,19 \pm 3,93$ & $-9,987$ & $<, 001$ & ,75 \\
\hline NDSS-Continuidad (tabaco/cigarrillo electrónico)a & $23,13 \pm 3,91$ & $25,90 \pm 4,86$ & $-2,819$ &, 006 & ,30 \\
\hline NDSS-Estereotipia (tabaco/cigarrillo electrónico)a & $11,64 \pm 2,95$ & $13,21 \pm 3,47$ & $-2,191$ &, 031 &, 24 \\
\hline
\end{tabular}

Nota. La dependencia de la nicotina entre los usuarios de cigarrillos electrónicos se evaluó usando las puntuaciones obtenidas de los cuestionarios para la evaluación de la dependencia del uso de los cigarrillos electrónicos; $\mathrm{CO}=$ monóxido de carbono; $\mathrm{ppm}=$ partes por $\mathrm{millón} ; \mathrm{ng} / \mathrm{mL}=\mathrm{nanogramo} / \mathrm{mililitro}$. ${ }^{\mathrm{a}}=\mathrm{Medias} \pm \mathrm{SD} ; t$ $=$ prueba t de Student; $r$ = tamaño de efecto $d$ de Cohen; FTND = Test de Fagerström de dependencia de la nicotina; NDSS = Escala del Síndrome de Dependencia de la Nicotina. 


\section{Fuentes de financiación}

te trabajo ha sido financiado por la Fundación BBVA (SV-14-FBBVA-1). Dicha entidad no asumió papel alguno en el diseño del estudio o en la recolección, el análisis o la interpretación de los datos, en la redacción del manuscrito, o en cualquier decisión relacionada con la presentación del manuscrito para su publicación.

\section{Conflicto de intereses}

Los autores declaran la inexistencia de conflictos de intereses.

\section{Referencias}

Cobb, C. O., Hendricks, P. S. y Eissenberg, T. (2015). Electronic cigarettes and nicotine dependence: evolving products, evolving problems. BMC Medicine, 13, 119. doi:10.1186/s12916-015-0355-y.

Foulds, J., Veldheer, S., Yingst, J., Hrabovsky, S., Wilson, S. J., Nichols, T. T. y Eissenberg, T. (2015). Development of a questionnaire for assessing dependence on electronic cigarettes among a large sample of ex-smoking E-cigarette users. Nicotine E् Tobacco Research, 17(2), 186-192. doi:10.1093/ntr/ntu204.

Harrell, P. T., Simmons, V. N., Correa, J. B., Padhya, T. A. y Brandon, T. H. (2014). Electronic nicotine delivery systems ("e-cigarettes"): review of safety and smoking cessation efficacy. Otolaryngology Head Neck Surgery, 151(3), 381-393. doi:10.1177/0194599814536847.

Heatherton, T. F., Kozlowski, L. T., Frecker, R. C. y Fagerstrom, K. O. (1991). The Fagerstrom Test for Nicotine Dependence: a revision of the Fagerstrom Tolerance Questionnaire. British Journal of Addictions, 86, 11191127.

Marsot, A. y Simon, N. (2015). Nicotine and Cotinine Levels with Electronic Cigarette: A Review. International Journal of Toxicology. doi:10.1177/1091581815618935.

Martínez-Sánchez, J. M., Ballbè, M., Fu, M., Martín-Sánchez, J. C., Saltó, E., Gottlieb, M.,... Fernández, E. (2014). Electronic cigarette use among adult population: a cross-sectional study in Barcelona, Spain (2013-2014). BMJ Open, , 4, e005894. doi:10.1136/bmjopen-2014-005894.

McMillen, R. C., Gottlieb, M. A., Shaefer, R. M., Winickoff, J. P., y Klein, J. D. (2015). Trends in Electronic Cigarette Use Among U.S. Adults: Use is Increasing in Both Smokers and Nonsmokers. Nicotine E Tobacco Research, 17, 1195-1202. doi:10.1093/ntr/ntu213.

Organización Mundial de la Salud. (2014). WHO report on the electronic nicotine delivery systems, 2014: Conference of the parties to the WHO framework convention on tobacco control. Retrieved at http://apps.who.
int/gb/fctc/PDF/cop6/FCTC COP6 10- en.pdf, July 8, 2016.

Shiffman, S., Waters, A. y Hickcox, M. (2004). The nicotine dependence syndrome scale: a multidimensional measure of nicotine dependence. Nicotine $\mathcal{E} 2$ Tobacco Research, 6, 327-348. doi:10.1080/1462220042000202481.

Yu, V., Rahimy, M., Korrapati, A., Xuan, Y., Zou, A. E., Krishnan, A. R.,... Ongkeko, W. M. (2016). Electronic cigarettes induce DNA strand breaks and cell death independently of nicotine in cell lines. Oral Oncology, 52, 58-65. doi:10.1016/j.oraloncology.2015.10.018. 